\title{
Uterine leiomyosarcoma: an unusual presentation
}

\section{Preethi B., Monu Singh*, Sarita Singh, Pallavi Singh}

Department of Obstetrics and Gynaecology, Safdarjung Hospital and VMMC, Delhi, India

Received: 05 June 2020

Accepted: 07 July 2020

\section{*Correspondence:}

Dr. Monu Singh,

E-mail: tamanna140690@gmail.com

Copyright: (c) the author(s), publisher and licensee Medip Academy. This is an open-access article distributed under the terms of the Creative Commons Attribution Non-Commercial License, which permits unrestricted non-commercial use, distribution, and reproduction in any medium, provided the original work is properly cited.

\section{ABSTRACT}

Uterine leiomyosarcomas are rare aggressive tumors, with high recurrence rates, even when confined to the uterine corpus at the time of diagnosis. It arises from smooth muscle of uterus and is a rare tumor that accounts for $2-5 \%$ of all uterine malignancies. These tumors typically spread hematogenously. Patients present with vague symptoms similar to those of patients with leiomyomas. Most patients are diagnosed with leiomyosarcoma postoperatively. Although prognosis remains dismal, various ongoing studies are investigating the role of advanced imaging, multimodality treatment, prognostic nomograms, and unique biomedical pathways to increase understanding of leiomyosarcoma and improve therapeutic options for patients. 46 years old para2 live2 postmenopausal (since 1.5 year) female presented to outpatient clinic with complaints of bleeding per vaginum, foul smelling discharge, unquantified weight loss and something coming out of vagina since 1.5 months. On abdominal examination, an irregular midline mass arising from pelvis corresponding to 20 weeks gestational size of uterus was present. On vaginal examination, 3 infected vaginal growths were present in vagina maximum $4 \times 4 \mathrm{~cm}$. Intra-operatively, uterus was nearly 20 weeks size with irregular surface. A $3 \times 3 \mathrm{~cm}$ subseroal fibroid with necrotic surface was present on posterior wall of uterus. Cut section of the operative specimen showed myohyperplasia which was compressing the uterine cavity, some necrotic areas were also present. Vaginal growths $-4 \times 4 \mathrm{~cm}$ on left vaginal wall near introitus with necrotic surface, $3 \times 3 \mathrm{~cm}$ on right vaginal wall, $1 \times 1 \mathrm{~cm}$ on right upper vaginal wall present. Excision of vaginal growth was done and was sent for histopathology. Histopathologic examination of sections of uterus showed all features were suggestive of leiomyosarcoma uterus.

Keywords: Chemotherapy, Radiotherapy, Salpingoophorectomy, Staging laparotomy, Uterine leiomyosarcoma

\section{INTRODUCTION}

Uterine leiomyosarcomas are rare aggressive tumors, with high recurrence rates, even when confined to the uterine corpus at the time of diagnosis. It arises from smooth muscle of uterus and is a rare tumor that accounts for $2-5 \%$ of all uterine malignancies. These tumors typically spread hematogenously. Patients present with vague symptoms similar to those of patients with leiomyomas. Most patients are diagnosed with leiomyosarcoma postoperatively. Although prognosis remains dismal, various ongoing studies are investigating the role of advanced imaging, multimodality treatment, prognostic nomograms, and unique biomedical pathways to increase understanding of leiomyosarcoma and improve therapeutic options for patients. ${ }^{1,2}$

\section{CASE REPORT}

A total 46 years old para2 live2 postmenopausal (since 1.5 year) female presented to outpatient clinic with complaints of bleeding per vaginum, foul smelling discharge, un-quantified weight loss and something coming out of vagina since 1.5 months.

On examination, vitals were stable. She was average built. On abdominal examination, an irregular midline mass arising from pelvis corresponding to 20 weeks 
gestational size of uterus was present. Lower margin could not be ascertained. The mass was firm to hard in consistency, unrestricted mobility, nontender with no free fluid. There was no hepatosplenomegaly.

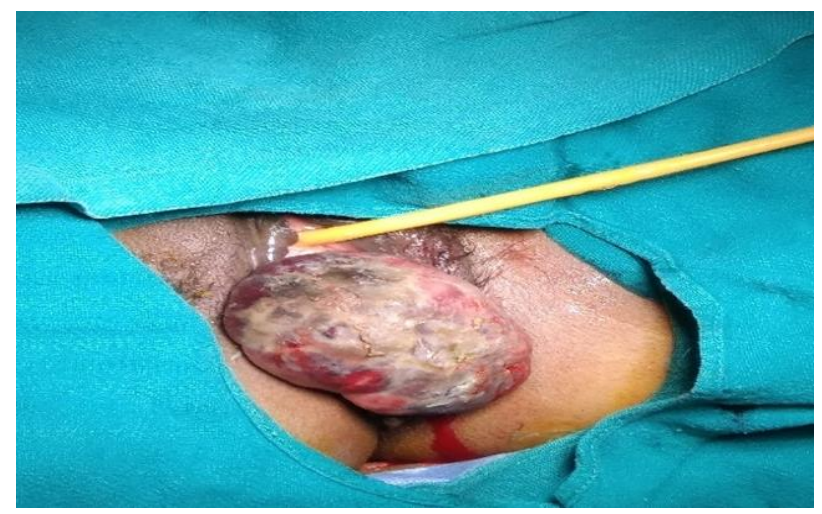

Figure 1: A vaginal growth protruding out of vagina.

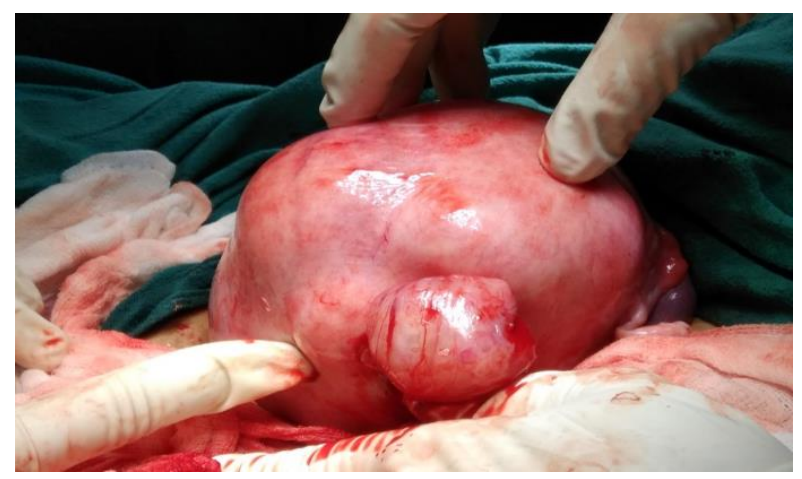

Figure 2: A subserosal fibroid on the posterior aspect of uterus.



Figure 3: Uterus with bilateral tubes and ovaries.

On vaginal examination, 3 infected vaginal growths were present in vagina maximum $4 \times 4 \mathrm{~cm}$ (Figure 1). Cervix could not be visualized on per speculum examination. On per vaginal examination uterus could not be felt.

On ultrasound uterus was anteverted, enlarged in size $16.9 \times 13.1 \times 10.2 \mathrm{~cm}$. Large ill-defined hypoechoic lesion size $13.3 \times 10.4 \times 4 \mathrm{~cm}$ and $5 \times 3 \times 3 \mathrm{~cm}$ seen in posterior uterine wall. Endometrial thickness $-4 \mathrm{~mm}$, bilateral ovaries normal. No free fluid in pouch of Douglas.
On CECT abdomen and thorax, uterus was significantly enlarged and extends superiorly up to the level of L3 vertebrae of size $10.1 \times 11.8 \times 13.5 \mathrm{~cm}$ and shows heterogenous enhancement with areas of necrosis. No calcification was seen. A large uterine fibroid with multiple subcentric lymph nodes present. Other results were normal. Patient underwent exploratory laparotomy followed by total abdominal hysterectomy with bilateral salpingoophorectomy with excision of the vaginal growth.

\section{Intra-op findings}

Intraoperatively, uterus was nearly 20 weeks size with irregular surface. A $3 \times 3 \mathrm{~cm}$ subseroal fibroid with necrotic surface was present on posterior wall of uterus (Figure 2, 3). Cut section of the operative specimen showed myohyperplasia which was compressing the uterine cavity, some necrotic areas were also present. Bilateral ovaries and tubes were normal in morphology. 3 vaginal growths, $4 \times 4 \mathrm{~cm}$ on left vaginal wall near introitus with necrotic surface, $3 \times 3 \mathrm{~cm}$ on right vaginal wall, $1 \times 1 \mathrm{~cm}$ on right upper vaginal wall present. Excision of vaginal growth was done and was sent for histopathology. Also $0.5 \times 0.7 \mathrm{~cm}$ skin tag near right vulva was excised and sent for histopathology.

Histopathologic examination of sections of uterus showed presence of a tumor arranged in the form of fascicles. The tumor shown high degree of cellular pleomorphism. The tumor cells are spindle in shape with moderate amount of eosinophilic cytoplasm and a hyperchromatic spindle shaped nucleus (Figure 4). Many atypical mitotic figure $3-4 / \mathrm{HPF}$ were seen with few areas of necrosis. All features were suggestive of leiomyosarcoma uterus. The bilateral adnexa were unremarkable. Vaginal growth on cut section showed presence of few hemorrhagic and necrotic areas with the same morphology as that of uterus. Sections of skin tag showed tumor of same morphology.

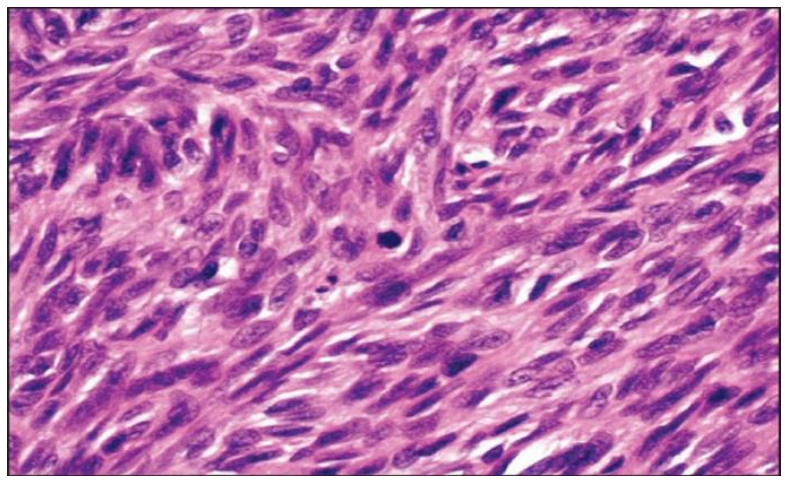

Figure 4: Cells are having hyperchromatic spindle shaped nuclei with dispersed chromatin, prominent nucleoli and prominent mitotic activity.

Immunohistochemistry - tumor cells were positive for SMA, desmin, vimentin and negative for CD10. 
Table 1: The staging of leiomyosarcoma is based on the FIGO classification of uterine sarcoma.

\begin{tabular}{|c|c|}
\hline \multicolumn{2}{|l|}{ Stage } \\
\hline Stage I & $\begin{array}{l}\text { Tumor limited to uterus } \\
(\mathrm{Ia}-<5 \mathrm{~cm}, \mathrm{Ib}>5 \mathrm{~cm})\end{array}$ \\
\hline Stage II & $\begin{array}{l}\text { Tumor extends to pelvis } \\
\text { (IIa-adnexal involvement, IIb - } \\
\text { extrauterine pelvic tissue involvement) }\end{array}$ \\
\hline Stage III & $\begin{array}{l}\text { Tumor invades abdominal tissue } \\
\text { (IIIa-one site, IIIb - >1 site } \\
\text { involvement in abdominal tissue, } \\
\text { IIIc - pelvic and para - aortic lymph } \\
\text { node involvement) }\end{array}$ \\
\hline Stage IV & $\begin{array}{l}\text { Tumor invades bladder, rectum and } \\
\text { distant metastasis }\end{array}$ \\
\hline
\end{tabular}

\section{DISCUSSION}

Leiomyosarcoma is one of the most common soft tissue sarcomas. It is classically considered that leiomyosarcomas are tumors that originate from the smooth muscle cells, or precursor mesenchymal stem cells committed to this line of differentiation. it is a subtype of uterine sarcoma. Uterine sarcoma can be divided into endometroid stromal sarcoma, undifferentiated and leiomyosarcoma. Leiomyosarcoma can arise in any soft tissue in the human body. Here authors discuss about the leiomyosarcoma arising in the uterus. $^{3}$

\section{Risk factors for uterine sarcoma}

These factors are known to change a woman's risk of developing a uterine sarcoma. ${ }^{2,3}$

- Pelvic radiation therapy - High-energy (ionizing) radiation used to treat some cancers can damage cells' DNA, sometimes increasing the risk of developing uterine sarcoma. These tumors generally develop 5 to 25 years later.

- $\quad$ Race - Uterine sarcomas are about twice as common in African-American women as they are in white or Asian women. The reason for this is unknown.

- $\quad$ RB gene changes - Women who have retinoblastoma due to abnormal copy of RB gene have an increased risk of uterine leiomyosarcomas.

\section{Signs and symptoms}

In most cases, the possibility of uterine sarcoma is suggested by certain symptoms but they don't always mean that a woman has uterine sarcoma. Still, these problems, need further evaluation and treatment. These symptoms include abnormal bleeding and spotting About $85 \%$ of patients diagnosed with uterine sarcomas have irregular vaginal bleeding (between periods) or bleeding after menopause. Some patients may also present with pelvic pain or pelvic mass. When they're first diagnosed, about $10 \%$ of women with uterine sarcomas have pelvic pain and/or a mass (tumor) that can be felt. Also, the patient may complain of fullness of stomach. ${ }^{2}$

\section{Diagnosis}

The first step in diagnosis of any medical condition is medical history, and complete physical examination including a pelvic examination. Following clinical examination various imaging modalities are used to diagnose the condition which include ultrasound (transvaginal or transabdominal), CT scan, MRI and PET SCAN. Following these imaging tests endometrial tissue sampling and testing is of prime importance. ${ }^{3,4}$

\section{Endometrial tissue sampling and testing}

To find the cause of abnormal uterine bleeding, a small piece of tissue (a sample) is taken from endometrium and evaluated. The tissue can be removed by endometrial biopsy or by dilation and curettage (D and $C$ ). Often a hysteroscopy is done with the $\mathrm{D}$ and $\mathrm{C}$ and tissue is taken from suspected areas under direct vision. This can help in the diagnosis of many endometrial stromal sarcomas and undifferentiated sarcomas, but only a few of leiomyosarcomas can be detected as these cancers start in the muscle layer of the wall of the uterus. To be found by an endometrial biopsy or D and C, they need to have spread from the muscle layer to the inner lining of the uterus. In most cases, the only way to diagnose an LMS is by removing it with surgery. ${ }^{4}$

\section{Testing endometrial tissue}

Any tissue samples taken out are tested to see if cancer is present. If cancer is present then its type and grade is determined. The tissue may also be tested to see if the cancer cells have estrogen receptors and progesterone receptors. These hormone receptors are found on many endometrial stromal sarcomas. Cancers with estrogen receptors on the cells are more likely to grow in response to estrogen, while those with progesterone receptors often have their growth decreased by progesterone. Checking for these receptors helps predict which patients will benefit from treatment with hormonal therapy. Also, immunohistochemistry markers are done to confirm the diagnosis. 4

\section{Cystoscopy and proctoscopy}

If a woman has signs or symptoms that suggest involvement of bladder or rectum. cystoscopy and proctoscopy may be required for determine the extent of involvement and treatment. ${ }^{4}$

\section{Chest X-ray}

A regular (plain) X-ray of the chest should be routinely done to look for metastasis in the lungs. ${ }^{4}$ 


\section{Staging of leiomyosarcoma}

The staging of leiomyosarcoma is based on the FIGO classification of uterine sarcoma. ${ }^{4}$ In stage I tumor is confined to uterus, stage II extends to pelvis, stage III involves abdominal tissue and stage IV extends to bladder, rectum and distant metastasis. In stage Ia tumor size is less than $5 \mathrm{~cm}$ and in stage Ib tumor size is more than $5 \mathrm{~cm}$. In stage IIa there is adnexal involvement and in stage IIb extrauterine pelvic tissue involvement is there. In stage IIIa one site and in stage IIIb more than 1 site involvement in abdominal tissue is seen. In stage IIIc pelvic and para aortic lymph node involvement is seen (Table 1).

\section{Treatment modalities}

Surgery: Surgery is the main treatment for any form of uterine sarcoma. The goal of surgery is to remove all of the cancer. Which is generally accomplished by removing the entire uterus (hysterectomy) along with fallopian tubes and ovaries. Also, part of the vagina may also need to be removed. Some lymph nodes or other tissue may be taken out as well to see if the cancer has spread outside the uterus. The type of surgery performed depends on the stage of cancer, patient's overall health and age. Following surgery options are used in the treatment of leiomyosarcoma. ${ }^{4,5}$

\section{Simple hysterectomy}

This surgery removes the whole uterus (the body of the uterus and the cervix). In a simple hysterectomy, the loose connective tissue around the uterus (called the parametrium), the tissue connecting the uterus and sacrum (the uterosacral ligaments), and the vagina are not removed. ${ }^{4,5}$

\section{Bilateral salpingo-oophorectomy}

This operation removes both fallopian tubes and both ovaries. In treating uterine leiomyosarcoma, this operation is usually done at the same time the uterus is removed. ${ }^{4,5}$

\section{Radical hysterectomy}

This operation removes the entire uterus along with the tissues next to the uterus and cervix (parametrium and uterosacral ligaments) and the upper part (about 1 inch) of the vagina (near the cervix). Such a radical surgery is performed only for complete removal of tumor tissue. ${ }^{4,5}$

\section{Lymph node surgery}

In cases where the tumor has spread outside the uterus or nearby lymph nodes look swollen on examination per operatively or where the imaging tests have shown enlarged lymph node lymph node dissection is performed, which removes lymph nodes in the pelvis and around the aorta (para-aortic). These lymph nodes are then checked under a microscope to see if they have cancer cells. If cancer is found in the lymph nodes, it means that the tumor has already spread outside of the uterus and further treatment with adjuvant therapy is needed. ${ }^{4,5}$ In cases where the diagnosis is in doubt staging laparotomy is performed for diagnosis and staging of the tumor.

\section{Other procedures done with the main surgery}

\section{Omentectomy}

Tumor sometimes spreads to the omentum, so during surgery omentectomy is performed. or sometimes it is removed as a part of staging surgery. ${ }^{4,5}$

\section{Peritoneal biopsies}

The tissue lining the pelvis and abdomen is called the peritoneum. Peritoneal biopsies remove small pieces of this lining to check for tumor cells as a part of staging laparotomy. ${ }^{4,5}$

\section{Pelvic washings}

In this procedure, the surgeon "washes" the abdominal and pelvic cavities with salt water (saline) and then sends the fluid to the lab to see if it contains cancer cells. ${ }^{4,5}$

\section{Tumor debulking}

If cancer has spread throughout the abdomen, the aim is to remove as much of the tumor as possible. This is called debulking. For some types of cancer, debulking can help other treatments (like radiation or chemotherapy) work better. Its role in treating uterine sarcoma or leiomyosarcoma isn't clear. ${ }^{4,5}$

\section{Radiation therapy}

Radiation therapy uses high-energy radiation (such as $\mathrm{x}$ rays) to kill cancer cells. Two types of radiation treatments may use for uterine sarcomas.

- External beam radiation therapy

- Internal radiation therapy or brachytherapy.

Sometimes both brachytherapy and external beam radiation therapy are used. How much of the pelvis needs to be exposed to radiation therapy and the type(s) of radiation used depend on the extent of the disease. ${ }^{5,6}$

\section{Role of radiation therapy in treating uterine sarcoma.}

- When the tumor can be seen growing through the cervix, radiation therapy might be used before surgery to make it easier to remove all the cancer 
- After surgery it may help lower the chance of tumor recurrence in the pelvis. This is called adjuvant radiation. It may be done for cancers that are high grade or when cancer cells are found in the lymph nodes. In these cases, the entire pelvis may be treated with external beam radiation therapy

- It may be the main treatment in a woman who can't have surgery because of other health problems

- It may be used to treat problems caused by tumor growth. For instance, radiation can be used to shrink a tumor that's causing pain and pressure symptoms. ${ }^{5,6}$

\section{Chemotherapy}

Chemotherapy can be given in the form of neo adjuvant (before surgery to shrink tumor size) or adjuvant therapy (if given after the cancer has been removed with surgery).

Some chemotherapeutic agents used in treating leiomyosarcoma are

- Dacarbazine (DTIC)

- Docetaxel (Taxotere $\left.{ }^{\circledR}\right)$

- Doxorubicin (Adriamycin $\left.{ }^{\circledR}\right)$

- Liposomal doxorubicin (Doxil®)

- $\quad$ Epirubicin (Ellence®)

- Gemcitabine (Gemzar®)

- Ifosfamide (Ifex $\left.{ }^{\circledR}\right)$

- Paclitaxel (Taxol®)

- Temozolomide (Temodar®)

- Trabectedin (Yondelis®)

- Vinorelbine (Navelbine®).

In most cases, more than one drug is used. For example, gemcitabine and docetaxel are often used together to treat leiomyosarcoma. ${ }^{2,6}$

\section{Hormone therapy}

Hormone therapy is mainly used to treat women with endometrial stromal sarcomas (ESS) and is rarely used for the other types of uterine sarcomas and leiomyosarcomas. ${ }^{7}$

\section{Targeted therapy for uterine sarcomas}

Targeted therapy is treatment with drugs that are made to target changes in the cancer cells. Targeted therapies only target cancer cells and leave healthy cells alone. They often cause fewer and different side effects than chemo. They are very new in the treatment of certain types of uterine sarcoma. Pazopanib (Votrient) is a targeted therapy that may be used to treat leiomyosarcoma that has spread or come back after treatment. ${ }^{7}$

\section{Stage wise treatment for leiomyosarcoma}

Surgery is the main treatment for all uterine sarcomas (Table 1). Followed by treatment with radiation, chemotherapy (chemo), or hormone therapy. Targeted therapy may also be used in advanced cancers. Women who can't have surgery because they have other health problems are treated with radiation, chemo, or hormone therapy. Often some combination of these treatments is used. Because uterine sarcoma is rare, it's has been hard to study it well. ${ }^{5,6}$

\section{Stages I and II}

Hysterectomy with bilateral salpingo-oophorectomy is done. with pelvic and para-aortic lymph node dissection or laparoscopic lymph node sampling may be done if swollen nodes are seen on imaging. During surgery, all pelvic and abdominal organs and the peritoneal lining are checked to see if the cancer has spread beyond the uterus. 5,6

Women with stage I cancers may not need more treatment and are put on follow up. In other cases, treatment with radiation, with or without chemo, may be needed after surgery if there's a high chance of the cancer coming back in the pelvis. This is called adjuvant treatment. For leiomyosarcoma of the uterus, adjuvant radiation lowers the chance of the cancer growing back in the pelvis (called local recurrence), but it doesn't affect the survival rates. Since the cancer can still come back in the lungs or other distant organs, some experts recommend giving chemo after surgery (adjuvant chemotherapy) for stage II cancers. ${ }^{5-7}$

\section{Stage III}

Surgery is done to remove all of the cancer. This includes hysterectomy, bilateral salpingo-oophorectomy, and lymph node dissection or sampling. If the tumor has spread to the vagina, part or even all of the vagina is removed.

After surgery, treatment with radiation (with or without chemo) may be offered to lower the chance of recurrence. Women who are very old or unfit for surgery may be treated with radiation and/or chemotherapy primarily. ${ }^{5,6}$

\section{Stage IV}

This is divided into stage IVA and stage IVB.

\section{Stage IVA}

Treatment includes complete removal with surgery if possible. If the cancer cannot be removed completely, radiation may be given, either alone or with chemotherapy. ${ }^{5}$

\section{Stage IVB}

There's no standard treatment for these cancers. Chemotherapy may be able to shrink the tumors for a time, but is not thought to be able to cure the cancer. 
Radiation therapy, given along with chemo, may also be an option. Also, targeted therapy may be tried. ${ }^{5}$

\section{Recurrent cancer}

Treatment options for recurrent uterine sarcoma are the same as those for stage IV. If the cancer can be removed, surgery may be done. If not already given, radiation or chemo may be given. .,7 $^{-1}$

\section{CONCLUSION}

Because of their rarity, uterine sarcomas are not suitable for screening. Surgery is the only treatment. The prognosis for women with uterine sarcoma primarily depends on the extent of disease and mitotic index. Due to their rarity, there is limited research on optimal management of this disease. Differentiating leiomyosarcoma from leiomyoma is challenging job and crucial for optimal treatment of the condition. During treating leiomyoma, the possibility of leiomyosarcoma should be kept in mind as a rare possibility and treatment options should be readily available.

Funding: No funding sources Conflict of interest: None declared

Ethical approval: Not required

\section{REFERENCES}

1. American Joint Committee on Cancer. Corpus Uteri. In: AJCC Cancer Staging Manual. $7^{\text {th }}$ ed. New York, NY: Springer; 2010: 403-409.

2. Bodurka DC, Gershenson DM. Sarcomas of the female reproductive tract. In: Pollock RE, ed.
American Cancer Society Atlas of Clinical Oncology. Soft Tissue Sarcomas. Hamilton, Ontario: BC Decker; 2002:213-27.

3. Chan JK, Kawar NM, Shin JY, Osann K, Chen LM, Powell CB, et al. Endometrial stromal sarcoma: a population-based analysis. $\mathrm{Br} \mathrm{J}$ Cancer. 2008;99:1210-5.

4. Cui, RR, Wright, JD, Hou, JY. Uterine leiomyosarcoma: a review of recent advances in molecular biology, clinical management and outcome. BJOG. 2017;124:1028-37.

5. Fields A, Jones JG, Thomas GM, Runowicz CD. Gynecologic cancer. In: Lenhard RE Jr, Osteen RT, Gansler T, eds. Clinical Oncology. Atlanta, Ga: American Cancer Society; 2001:455-96.

6. Francis JH, Kleinerman RA, Seddon JM, Abramson DH. Increased risk of secondary uterine leiomyosarcoma in hereditary retinoblastoma. Gynecol Oncol. 2012;124(2):254-9.

7. Freedman DM, Curtis RE, Travis LB, Fraumeni Jr JF. New Malignancies Following Cancer of the Uterine Corpus and Ovary. In: Curtis RE, Freedman DM, Ron E, Ries LAG, Hacker DG, Edwards BK, Tucker MA, Fraumeni JF Jr. (eds). New Malignancies Among Cancer Survivors: SEER Cancer Registries, 1973-2000. National Cancer Institute. NIH Publ. No. 05-5302. Bethesda, MD, 2006.

Cite this article as: Preethi B, Singh M, Singh S, Singh P. Uterine leiomyosarcoma: an unusual presentation. Int J Reprod Contracept Obstet Gynecol 2020;9:3493-8. 